\title{
Computational Interaction Analysis of Cyprinus carpio Trypsin1 and Kunitz Type Soybean Protease Inhibitor
}

\author{
Gulshan Kumar*, Munish Kumar, Gyandeep Gupta and Ranjeeta Kumari
}

Division of Genetics and Biotechnology, ICAR-Central Institute of Fisheries Education, Versova - 400 061, Mumbai, India

*Corresponding author

\begin{tabular}{|c|}
\hline Keywords \\
\hline $\begin{array}{l}\text { Cyprinus carpio, } \\
\text { Aquaculture, } \\
\text { Protease inhibitor, } \\
\text { Trypsin, Soybean } \\
\text { meal, computational } \\
\text { modelling }\end{array}$ \\
\hline Article Info \\
\hline $\begin{array}{l}\text { Accepted: } \\
24 \text { May } 2019 \\
\text { Available Online: } \\
\text { 10 June } 2019\end{array}$ \\
\hline
\end{tabular}

Common carp (Cyprinus carpio) is highly important culturable species in freshwater and cultured throughout the globe. Its farming require formulated feed with balance protein and energy. Most of the time fish meal is used as a protein source, but it is not acceptable from sustainability point of view. The replacement of fish meal by plant based protein source is advised. The limitation of plant based ingredients like soybean meal is the presence of anti-digestive factors in the form of several protease inhibitors. Kunitz type soybean protease inhibitor (SPI) is the most abundant protease inhibitor in soybean meal and it reduces the digestibility of feed. The data regarding molecular interaction and binding affinity of SPI with proteases is available in mammalian organism like pig but in fishes it has not been studied in detail. So the present study was conducted to gain insight into the protein-protein interaction and binding affinity of common carp trypsin (ccTrypsin1) and SPI using computational modelling. The sequence and 3D structure of ccTrypsin 1 is highly similar to its mammalian counterpart pig. The protein-protein interaction analysis using knowledge based docking approach showed that the interaction was more or less similar like porcine trysin with SPI. The predicted binding affinity $(-9.2$ $\mathrm{kcal} / \mathrm{mol}$ ) and dissociation constant (1.9E-0.7) showed strong interaction between the interactor governed by strong attractive forces like electrostatic forces.

\section{Introduction}

Aquaculture is the fastest growing food producing sector in the world. Global per capita fish availability has reached $20 \mathrm{~kg}$, which is achieved by the significant increase in the aquaculture production both from freshwater and brackish water (SOFIA, 2016). The Asian continent is predominant in aquaculture production, which includes many species like carps, catfishes, shellfish, pangasius, tilapia etc. The three Indian major carp (IMCs), namely catla (Catla. catla), rohu (Labeo. rohita) and mrigal (Cirrhinus. mrigala) contribute the bulk of production to the extent of 70 to $75 \%$ of the total freshwater fish production, followed by exotic carps comprising silver carp, grass carp and common carp forming the second important group contributing to the balance 25 to $30 \%$ (FAO, 2018). Among exotic carps, the Cyprinus carpio is the important cyprinid 
species for aquaculture in many Asian and some European countries (Rahman, 2015) and third most widely cultivated freshwater species contributing $8 \%$ to the aquaculture in the world (FAO, 2018). It is a hardy fish that thrive in a wide variety of aquatic habitats and also tolerates a wide range of temperatures (3.0-35 ${ }^{\circ} \mathrm{C}$ ) (Froese and Pauly, 2011), salinity up to $9 \mathrm{ppt}, \mathrm{pH}$ (6.5-9.0) and dissolved oxygen concentration (0.3-0.5 mg L-1) as well as at supersaturation of oxygen level (Azizi et al., 2011).

The practice of semi-intensive and intensive culture system has shifted the carp culture from the non-feed based extensive systems to supplementary or complete feed based culture system. In finfish aquaculture, feed cost is the main expenditure as fishmeal is used as the protein source for the diet. Over the last 20 years, fish nutrition research has focused on the replacement of fishmeal with more sustainable alternative protein ingredients (Bowyer et al., 2013; Tacon and Metian, 2008). It was identified that alternative protein ingredients should possess adequate nutritional properties, that is a high level of protein with a favorable amino acid profile, high nutrient digestibility and acceptable palatability, and be relatively inexpensive compared with fishmeal (Gatlin et al., 2007).

Soybean meal is currently the most common plant protein used as a fishmeal replacer and has been used as a feed ingredient for a variety of fish species due to its high content of available essential fatty acids and unsaturated fatty acids, and for its favorable amino acid profile (Hertrampf and Piedad- Pascual, 2000). However, to date, soybean meal usually makes up approximately $20 \%$ of the fish feed ingredient. A higher level of soybean meal could reduce fish growth, feed intake and digestibility because of the presence of antinutritional factors such as trypsin inhibitor, phytates, lectins and saponins in the soybean meal (NRC, 2011).

Legume seeds protease inhibitors (PIs) are usually inhibitors of serine proteinases and exhibit strong inhibitory activity against trypsin, chymotrypsin or both. Inhibitory activity against proteinases of other mechanistic classes such as cysteine- or metallo-proteinases is rarely observed. Biochemical characterization of these inhibitor proteins resulted in identification of two subtypes, viz., Bowman-Birk-type (BBI) and Kunitz-type; these subtypes have similar specificities, but they differ in their biochemical and physical properties. The Bowman-Birk type inhibitors were initially described in soybeans (Glycine max) as inhibitors of trypsin (Bowman, 1946; Birk, 1963) and subsequently reported with dual specificity towards trypsin and chymotrypsin in soybeans (Birk et al., 1961; Birk, 1985), as well as other legumes like chickpeas ( $C$. arietinum) (Belew et al., 1975; Belew and Eaker, 1976; Smirnoff et al., 1976; Jibson et al., 1981; Birk, 1985) are single chain polypeptides of 6-12 kDa and possess seven intra-chain disulfide bridges.

Kunitz-type PIs are single chain polypeptides of $\sim 20 \mathrm{kDa}$, characterized by presence of two intra-chain disulfide bridges, and usually having a single activity site (inhibitory loop) although secondary activity has also been reported (Franco et al., 2002). Owing to the single active site, these inhibitors bind to proteinases in a simple 1:1 fashion. At the molecular level, Kunitz type PIs has a roughly spherical shape and the structure is characterized as a ' $\beta$-trefoil fold'. The amino acids responsible for serine proteinase binding and inhibition are located on an extended 'binding' loop, which is structurally similar across the Kunitz-type PIs. The specificity towards target proteinase is determined by the 
nature of the amino acid residue at the PI position on the 'binding' loop - basic sidechain amino acids like arginine or lysine are usually associated with trypsin specificity whereas hydrophobic amino acids (phenylalanine>others) are linked to chymotrypsin specificity. Legume PIs follow the classical mechanism of proteinase inhibition, i.e., the inhibitory loop of the PI mimics the substrate and binds to the protease, forming a stable complex, thus inactivating the protease (Franco et al., 2002).

Data regarding molecular interaction and binding affinity of species specific proteases with protease inhibitor is not available. Computational prediction of three dimensional structure and binding affinity can give us valuable insights regarding molecular mechanism and strength of interaction. These data can be valuable for feed formulation and ingredient selection for common aquaculture species. Present study was designed to predict three dimensional structure of Cyprinus carpio trypsin 1 (ccTrypsin1) and its interaction with Kunitz type soybean protease inhibitor (SPI).

\section{Materials and Methods}

\section{Sequence retrieval and analysis}

Sequences of ccTrypsin1 (Accession no. BAL04385.1) and porcine pancreatic trypsin PDB ID (1AVW) were retrieved from NCBI and PDB database respectively for comparison. Both sequences were aligned using Clustal Omega program (https://www. ebi.ac.uk/Tools/msa/clustalo/) with default settings. Clustal Omega (Chojnacki et al., 2017) is a multiple sequence alignment program that uses seeded guide trees and HMM profile-profile techniques to generate alignments 3D structure of SPI (POB ID 1BA7.A) was downloaded from PDB database.

\section{Homology modeling}

Swiss-Model (Guex et al., 2009; Waterhouse et al., 2018) (www.expasy.ch/ swissmod/SWISS-MODEL.html) is an automated modeling server that allows an user to submit a sequence and to get back a structure automatically. It identifies template using BLAST and HHbits. After selection of suitable templates, a raw model is built based on rigid fragment assembly approach. Refinement of the structure is done using GROMOS (www.igc.ethz.ch/gromos/). The server uses OpenMM library to perform the computation and CHARMM27 for parameterization. Three dimensional structure of ccTrypsin 1 was built using this server in automatic alignment mode with server defined template. Predicted structure was energy minimized by SWISS-MODEL server itself with default settings.

\section{Model validation}

The quality of the structure generate was evaluated using Ramachandran plot analysis and QMEAN (Benkert et al., 2011) (https://swissmodel.expasy.org/qmean/)

method. Local quality of the model was evaluated based on QMEANDisco (Waterhouse et al., 2018) Ramachandran plot displays the geometry of the structure by showing residue wise torsion angles. Procheck (Laskowski et al., 1993) tool was used for Ramachandran plot analysis. It gives total number and percentage of the residues distributed in allowed and disallowed regions of the plot.

QMEAN is a composite estimate based on geometrical properties and provides both global and local quality of the structure. On global scale QMEAN Z-score tells about the nativeness of the structure. In other words it tells that whether the model is comparable in quality with experimentally determined 
structure of same size. Local quality of the model is given in terms of residue-wise local quality score which denotes again the nativeness of geometrical properties of individual amino acids compared with experimentally determined structures.

\section{Structural superimposition}

Matchmaker tool of UCSF-Chimera (Pettersen et al., 2004) software was used for superimposition of structures. ccTrypsin 1 structure was superimposed to porcine structure (1AVW chain A). The alignment algorithm used was Needleman-Wunsch with BLOSUM-62 matrix. Matching was iterated by pruning long atom pairs until no atom pairs exceeds $2.0 \AA$.

\section{Docking of ccTrypsin 1 with SPI}

Docking of ccTRypsin1 with SPI was performed using HADDOCK (High Ambiguity Driven Protein-protein Docking) server (Van Zundert et al., 2016) (https://milou.science.uu.nl/services/HADDO CK2.2/haddock.php). Easy interface with default settings was applied for docking. Amino acid residues involved in interaction was supplied based on Clustal alignment of the sequences. HADDOCK server performs data-driven flexible docking approach for docking of proteins. User has to supply some of the amino acids present at the binding interface of both the interacting proteins.

\section{Prediction of binding affinity and dissociation constant}

PRODIGY (Protein Binding Energy Prediction) server (Vangone and Bonvin, 2015; Xue et al., 2016) was used for prediction of binding energy and dissociation constant for ccTrypsin1-SPI complex. This server also provides list of pairing residues at the interface of interacting proteins. This server uses only structural information of the complex to determine binding affinity. The server uses two important criteria forming the basis of prediction which is number of interfacial contacts and non-interacting surface properties.

\section{Results and Discussion}

\section{Structural prediction}

Sequence alignment between porcine trypsin and ccTrypsin1 protein shows very high sequence conservation (Fig. 1). From this alignment residues coming at the interface between ccTrypsin 1 and SPI were also identified and used further for docking in next step. Three dimensional (3D) structure of ccTrypsin1 was generated using SWISSMODEL in monomeric state without any ligand.

The template taken was Salmosalar trypsin (PDB ID 1UTK.1.A). The sequence identity of the template with target viz. ccTrypsin 1 was $82.27 \%$ and query cover $91 \%$. The template structure was solved by $\mathrm{X}$-ray crystallography with resolution $1.53 \AA$ Amino acid residues $21-239$ of the target were modeled successfully. As part of PA clan superfamily ccTrypsin 1 structure was having double beta barrel arrangement (Fig. 2a). The arrangement of barrel was perpendicular forming the core of the structure. The core of the structure was surrounded by $\alpha$-helices and coils.

The 3Dstructure of ccTrypsin1 was made up of $12 \beta$-strands, $3 \alpha$-helices and several coils. This structure was perfectly superimposed to porcine structure (Fig. 2b). The core $\beta$-barrel and $\alpha$-helices were aligned perfectly. Some of the coils were deviated in the ccTrypsin 1 structure. Region containing amino acid Met145 to Ser151 was not aligned with each other. 


\section{Model validation}

Ramachandran plot analysis showed that $87.4 \%$ of the total residues were in most favored region and none of the residue was in disallowed region (Fig. 3 and Table 1). As the percentage of most favored residue was close to $90 \%$, the quality of 3D structure was acceptable. Global quality of the model was evaluated by QMEAN score given by SWISS MODEL server (Fig. 4a and b). The QMEAN value of the structure was 0.93 implying good quality of the overall structure. Local quality value of individual amino acids were also showed that most of the regions of the 3D structure were modeled with good quality. The region between the residue 145 to 155 were of low quality.

\section{Docking of ccTrypsin1 with SPI}

For data driven docking using HADDOCK the interacting residues from both structures viz. ccTrypsin 1 and PSI were specified to the server. For ccTrypsin His60, Ser98, Gln193 and Ser196 and for SPI, Asn13, Tyr62, Arg63 and Ilu64 were specified based on reports from porcine complex. HADDOCK clustered 172 structures in 7 clusters which represents $86 \%$ of water refined structures generated by the server. Cluster 1, the most populated cluster contained 69 structure with energy score -96.4+/-4.4 (Table 2). The lowest energy scoring cluster was cluster with docking energy score $-100+/-8.3$ with cluster size 12 . The best structure of the cluster 1 and 3 were superimposed to porcine complex $(1 \mathrm{AVW})$ to confirm the nativness of the predicted complex. As both clusters were having similar energy values and cluster 1 was closer towards native structure, this cluster was taken for further analysis. Significant energy terms contributing in interaction were electrostatic, Van der Waals and dissolvation energies for this cluster. In the predicted protein-protein complex, SPI covers the active site area of ccTrypsin 1 found at the interface of two $\beta$-barrels (Fig. $5 a)$. The loop region made from residue Ser60 to Phe66 projected from the $\beta$-core of the SPI makes contact to the ccTrypsin 1 active area. Some deviations from porcine complex was also observed after superimposition of the ccTrypsin-PSI complex to porcine complex (Fig. 5b). The above mentioned loop was deviated around $3.44 \AA$ (measured at Arg63 of PSI) away from active area than porcine structure The region of PSI facing away from ccTrypsin1 was even more deviated downward with a distance around $18.98 \AA$ when measured at Met84 of SPI.

Table.1 Ramachandran plot statistics from figure 1

\begin{tabular}{|l|c|c|}
\hline Parameters & $\begin{array}{c}\text { Number of Amino } \\
\text { acids }\end{array}$ & Percentage \\
\hline Residues in most favored regions [A,B,L] & 160 & $87.4 \%$ \\
\hline Residues in additional allowed regions [a,b,l,p] & 23 & $12.6 \%$ \\
\hline Residues in generously allowed regions [ a, $, \mathbf{b}, \sim \mathbf{l}, \sim \mathbf{p}]$ & 0 & $0.0 \%$ \\
\hline Residues in disallowed regions & 0 & $0.0 \%$ \\
\hline Number of non-glycine and non-proline residues & 183 & $100.0 \%$ \\
\hline Number of end-residues (excl. Gly and Pro) & 2 & \\
\hline Number of glycine residues (shown as triangles) & 24 & \\
\hline Number of proline residues & 10 & \\
\hline Total number of residues & 219 & \\
\hline
\end{tabular}


Table.2 Haddock score and energy values for selected cluster

\begin{tabular}{|l|c|}
\hline Parameters & Values \\
\hline HADDOCK score & $-96.4+/-4.4$ \\
\hline Cluster size & 69 \\
\hline RMSD from the overall lowest-energy structure & $6.0+/-0.2$ \\
\hline Van der Waals energy & $-54.8+/-3.7$ \\
\hline Electrostatic energy & $-65.8+/-32.6$ \\
\hline Dessolvation energy & $-28.6+/-3.7$ \\
\hline Restraints violation energy & $1.7+/-0.57$ \\
\hline Buried Surface Area & $1372.8+/-90.0$ \\
\hline Z-Score & -1.0 \\
\hline
\end{tabular}

Table.3 PRODIGY prediction details

\begin{tabular}{|l|c|}
\hline Parameters & Values \\
\hline Dissociation constant $\left(\mathbf{K}_{\mathbf{d}}\right)$ at $\mathbf{2 5}^{\circ}$ & $1.9 \mathrm{E}-07$ \\
\hline Binding affinity $(\boldsymbol{\Delta} \mathbf{G})$ & $-9.2 \mathrm{kcal} \mathrm{mol}^{-1}$ \\
\hline ICs charged-charged $*$ & 7 \\
\hline ICs charged-polar & 6 \\
\hline ICs charged-apolar & 20 \\
\hline ICs polar-polar & 1 \\
\hline ICs polar-apolar & 6 \\
\hline ICs apolar-apolar & 14 \\
\hline NIS charged** & $23.91 \%$ \\
\hline NIS apolar & $39.13 \%$ \\
\hline
\end{tabular}

*Interfacial contacts (IC), **Non-interacting surfaces (NIS)

Fig.1 Clustal alignment of ccTrypsin with porcine trypsin

1AVW: A porcine trypsin BAL04385.1 ccTrypsin1

1AVW:A porcine trypsin BAL04385.1 ccTrypsin1

1AVW: A porcine trypsin BAL04385.1 ccTrypsin1

1AVW:A porcine trypsin BAL04385.1 ccTrypsin1

1AVW: A porcine trypsin BAL04385.1 ccTrypsin1
MRSLVFLVLGAAFALDGDKIVGGYECTPHSQPWQVSLNSGYHFCGGSLVSEYWVSAAH
$* * * * * *::^{*} * * * * * * * * * * * * *: \ldots * * * * * *$
CYKSRIQVRLGEHNIDVLEGNEQFINAAKIITHPNFNGNTLDNDIMLIKLSSPATLNSRV
CYKSRVEVRLGEHNIVLNEGSEQFISSEKVIRHPNYNSWTIDSDIMLIKLSKPATLNQYV $* * * * *:: * * * * * * * *: * * . * * * * .: *^{*}: * * *: * . *$ : $^{*}, * * * * * * * * . * * * * * . *$

ATVSLPRSCAAAGTECLISGWGNTKSSGSSYPSLLQCLKAPVLSDSSCKSSYPGQITGNM 160 QPVALPSGCAADGTMCRVTGWGNTMSSTA-DSNKLQCVEVPILSERDCNNSYPGMITNTM 179

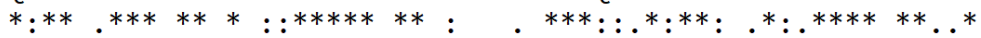

ICVGFLEGGKDSCQGDSGGPVVCNGQLQGIVSWGYGCAQKNKPGVYTKVCNYVNWIQQTI 220 FCAGYLEGGKDSCQGDSGGPVVCNGQLQGIVSWGYGCAEKNHPGVYGKVCMFSQWIADTM 239

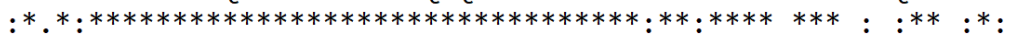

AAN 223

KNN 242 
a.

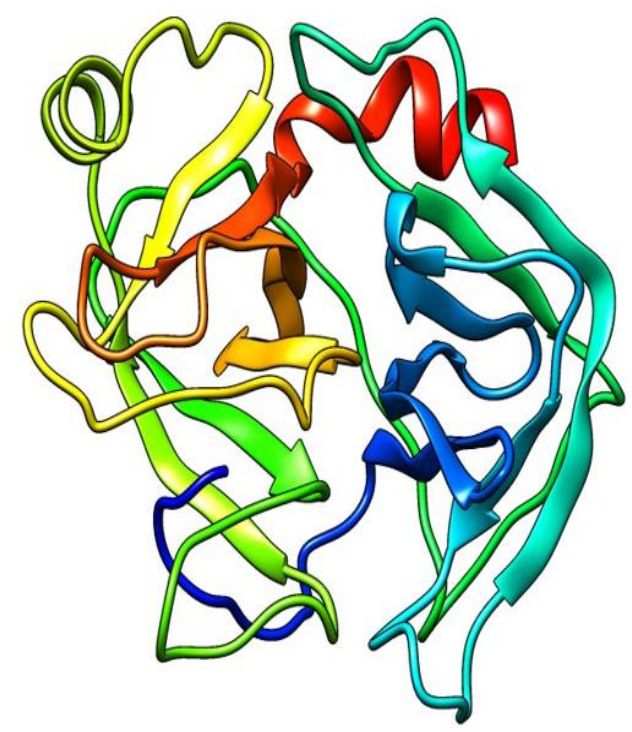

b.

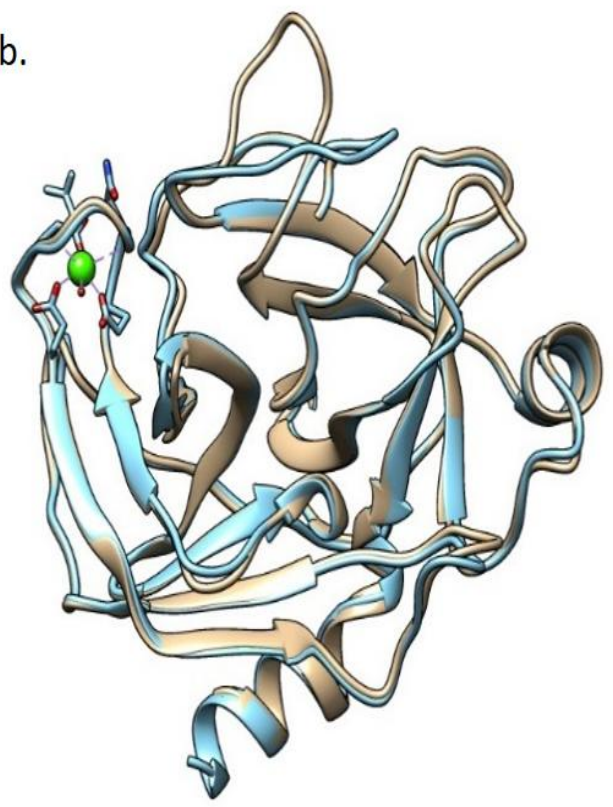

- Fig.2.a. 3D structure of ccTrypsin. Secondary structural elements are highlighted with different colures. b. ccTrypsin structure (golden) porcine trypsin (blue) were superimposed.

Fig.3 Ramachandran plot analysis of ccTrypsin1 structure

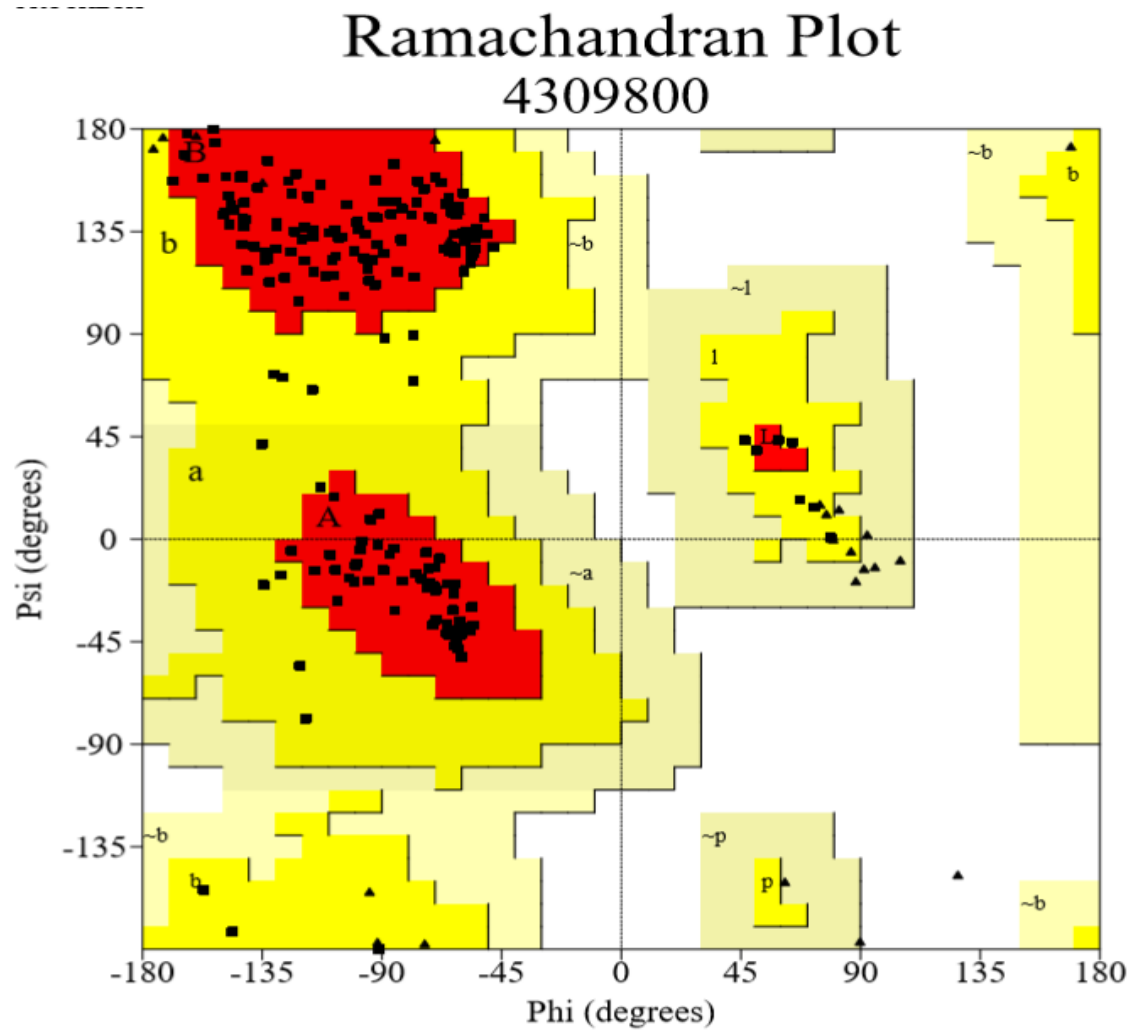


a.

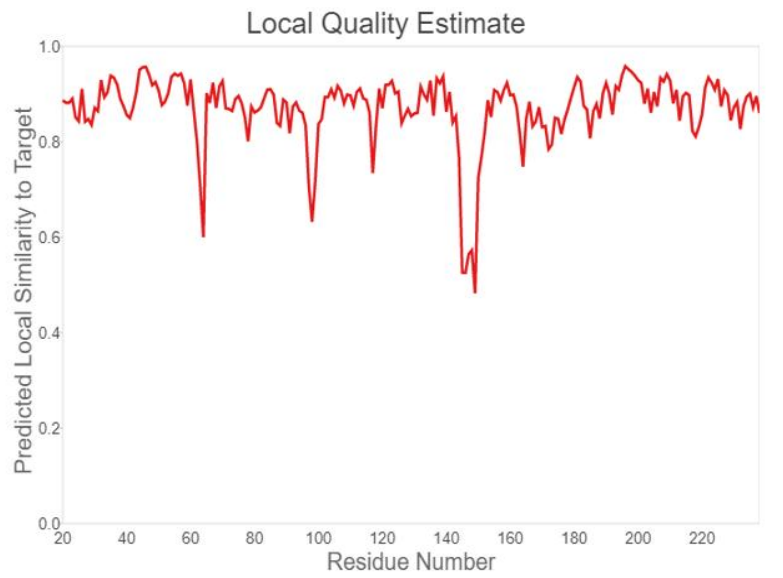

b.

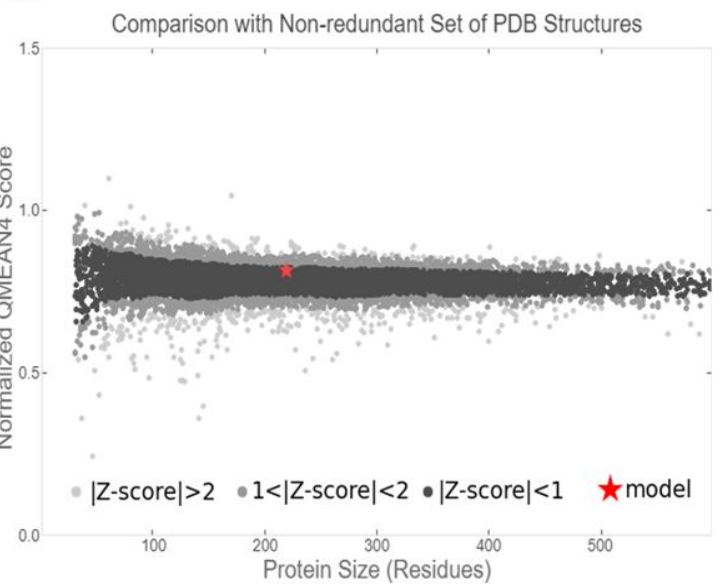

Fig. 4.a Local quality estimate 4 b. Global quality estimate
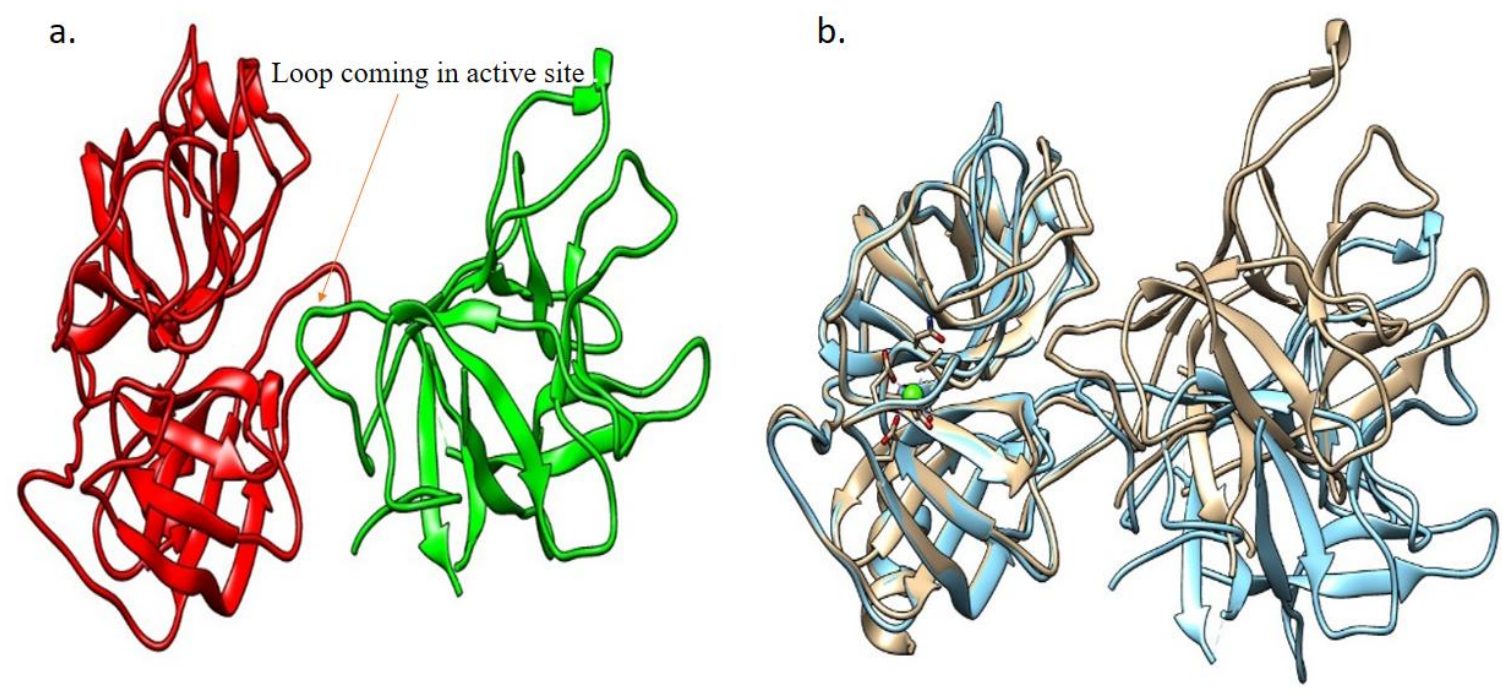

Fig. 5.a. ccTrypsin-SPI complex structure; ccTrypsin is coloured red and SPI is coloured green. b. ccTrypsin-PSI complexed (blue) was superimposed to porcine trypsin complexed with (golden) SPI (PDB ID. 1AVW)

\section{Prediction of binding energy and dissociation constant}

Binding energy $(\Delta \mathrm{G})$ and molar dissociation constant $\left(\mathrm{K}_{\mathrm{d}}\right)$ was predicted by PRODIGY server. The value of binding energy of the complex ccTrypsin1-SPI was $-9.2 \mathrm{kcal} / \mathrm{mol}$ and dissociation constant was $1.9 \mathrm{E}-0.7$ at $25^{\circ} \mathrm{C}$. Both charged and uncharged contacts contributed in binding energy. While charged- apolar contacts dominated, there was only one polar-polar contacts (Table 3). The contribution of non-interacting surface apolar property was higher than charged in binding energy value.

Trypsin is serine protease of PA clan superfamily. It is common in digestive systems of many vertebrates where it plays crucial role in protein digestion. Trypsin from 
different organism including fishes was shown to have very similar overall structure (Rawlings and Barrett, 1994; Di Cera, 2009). Among fishes there is high sequence conservation. The enzyme contains core domain made up of two $\beta$-barrels surrounded by $\alpha$-helices and loops. This arrangement is conserved throughout the superfamily. The active site of the enzyme is located between the interface of the two $\beta$-barrels ((Rawlings and Barrett, 1994; Di Cera, 2009). We also observed same structural fold in ccTrypsin 1 enzyme which is very obvious. Both sequence and overall structure of ccTryposin 1 was highly similar to well characterized porcine enzyme. Superimposition of the two 3D structure shows perfect alignment of secondary structures. The area near the active region was well aligned. Some loop regions away from active site were seen deviating. This implies the perfect conservation in the catalytic strategy and thus enzyme functioning in C. carpio.

Kunitz type soyabean protease inhibitor is a strong inhibitor of trypsin and can be hydrolyzed by trypsin. The loop containing Arg63 and Ile64 projects in the active site of the enzyme. These two amino acids forms the cleavage site for the enzyme (Ozawa and Laskowski, 1966; Song and Suh, 1998). Thus SPI covers the active area of enzyme and prevents access to other substrate (Ozawa and Laskowski, 1966; Song and Suh, 1998). In this study similar type interaction was observed where above mentioned residues come very close to active site of the enzyme. Binding affinity is a measure of strength of interaction between two interacting molecules. Dissociation constant is the experimental measure of binding affinity. Binding affinity is dependent on both chemical interaction and surface area involved in binding. The observed rate constants of binding between protein-protein complexes lie $<10^{3} \mathrm{M}^{-1} \mathrm{~s}^{-1}$ to $>10^{9} \mathrm{M}^{-1} \mathrm{~s}^{-1}$. The value in the range $10^{8}$ to $10^{9}$ as observed in many protein-protein complexes shows strong interaction and involves long range electrostatic interactions (Schreiber et al., 2009; Kastritis and Bonvin, 2013). In this study we also observed dissociation constant in this range showing strong interaction between ccTrypsin 1 and SPI. In this binding affinity there is also significant contribution of electrostatic interaction as seen in chargedcharged and charged-apolar contacts.

\section{Acknowledgements}

The authors are grateful to the Director, Central Institute of Fisheries Education, Mumbai, for providing facilities for carrying out the research work. The first author is grateful to the CIFE for the institutional support provided during the period of work.

\section{References}

Alexis, M. N., and Nengas, I., 2001. Current state of knowledge concerning the use of soy products in diets for feeding sea bass and sea bream needs for future research (pp. 1- 32). Brussels, Belgium.

Azizi, S., Kochanian, P., Peyghan, R., Khansari, A. and Bastami, K. D., 2011.Chloride cell morphometrics of Common carp, Cyprinus carpio, in response to different salinities. Comparative Clinical Pathology, 20 (4): 363-367.

Belew, M., Porath, J., Sundberg, L., 1975. The trypsin and chymotrypsin inhibitors in chickpeas (Cicer arietinum L). Purification and properties of the inhibitors. European Journal of Biochemistry 60: 247-258.

Benkert, P., Biasini, M. and Schwede, T., 2010. Toward the estimation of the absolute quality of individual protein structure models. Bioinformatics, 27(3), 343-350. 
https://doi.org/10.1093/bioinformatics/b tq662

Birk, Y., 1961. Purification and some properties of a highly active inhibitor of trypsin and alphachymotrypsin from soybeans. BiochimicaetBiophysicaActa 54: 378-381.

Birk, Y., 1985. The Bowman-Birk inhibitor. Trypsin- and chymotrypsin-inhibitor from soybeans. International Journal of Peptide and Protein Research. 25: 113 131.

Birk, Y., Gertler, A., Khalef, S., 1963. A pure trypsin inhibitor from soya beans. The Biochemical Journal 87: 281-284.Belew M and Eaker D (1976) The trypsin and chymotrypsin inhibitors in chickpeas (Cicer arietinum L). Identification of the trypsin-reactive site, partial-aminoacid sequence and further physicochemical properties of the major inhibitor. European Journal of Biochemistry 62: 499-508.

Bowman, D. E. 1946. Differentiation of soybean antitrypsin factors. Proceedings of the Society for Experimental Biology and Medicine 63: 547-550.

Bowyer, J. N., Qin, J. G., and Stone, D. A. J., 2013. Protein, lipid and energy requirements of cultured marine fish in cold, temperate and warm water. Reviews in Aquaculture, 5, 10 32.

Chojnacki, S., Cowley, A., Lee, J., Foix, A. and Lopez, R., 2017. Programmatic access to bioinformatics tools from EMBL-EBI update: 2017. Nucleic acid $s$ research, 45(W1), W550-W553. https://doi.org/10.1093/nar/gkx273

Di Cera, E., 2009. Serine proteases. IUBMB life, 61(5), pp.510-515.

Duranti, M., A. Barbiroli, A. Scarafoni, G. Tedeschi and P. Morazzoni., 2003. Onestep purification of Kunitz soybean trypsin inhibitor. Protein Expr. Purif., 30: 167-170.
FAO, 2018. The state of world fisheries and aquaculture - Meeting the sustainable development goals. Food and Agriculture Organization, Rome, 210.

Franco, O.L., Grossi, de Sa, M.F., Sales, M.P., Mello, L.V., Oliveira, A.S., Rigden, D.J., 2002. Overlapping binding sites for trypsin and papain on a Kunitz-type proteinase inhibitor from Prosopisjuliflora. Proteins 49: 335-341.

Froese, R. and Pauly, D., 2011.FishBase. World Wide Web electronic publication. Available at: http://www. Fish base. org.

Gatlin, D. M., Barrows, T. F., Brown, P., Dabrowski, K., Gaylord, T. G., Hardy, ～R. W., Wurtele, E., 2007. Expanding the utilization of sustainable plant products in aquafeeds: A Research, 38, 551- 579.

review. Aquaculture

Guex, N., Peitsch, M.C. and Schwede, T., 2009. Automated comparative protein structure modeling with SWISS-MODEL and Swiss- PdbViewer: A historical perspective. Electrophoresis, 30(S1), S162-S173. https://doi.org/10.1002/elps.200900140

Halver, J. E., and Hardy, R. W., 2002. Nutrient flow and retention. In J. E. Halver, \& R. W. Hardy (Eds.), Fish Nutrition. California, CA: Academic Press.

Hepher, B., 1988. Nutrition of pond fishes. Cambridge University Press.

Hertrampf, J. W., and Piedad- Pascual, F., 2000. Handbook on ingredients for aquaculture feeds. Dordrecht, the Netherlands: Kluwer Academic Publishers.

Iqbal, K.J., Ashraf, M., Abbas, F., Javid, A., Hafeez-ur-Rehman, M., Abbas, S. and Altaf, M., 2014. Effect of plantfishmeal and plant by-product based feed on growth, body composition and 
organoleptic flesh qualities of Labeorohita. Pakistan J. Zool., 46(1):253-260.

Jibson, M. D., Birk, Y., Bewley, T.A., 1981. Circular dichroism spectra of trypsin and chymotrypsin complexes with Bowman-Birk or chickpea trypsin inhibitor. International Journal of Peptide and Protein Research 18: 26-32.

Kastritis, P.L. and Bonvin, A.M., 2013. On the binding affinity of macromolecular interactions: daring to ask why proteins interact. Journal of The Royal Society Interface, 10(79), p.20120835.

Khan, M. A., Jafri, A. K., Chadha, N. K. and Usmani, N., 2004. Growth and body composition of rohu (Labeorohita) fed diets containing oilseed meals: partial or total replacement of fishmeal with soybean meal. Aqua. Nutr., 9:391-396.

Kunitz, M., 1945. Crystallization of a trypsin inhibitor from soyabean. Science 101: 668-669.

Laskowski, R.A., MacArthur, M.W., Moss, D.S. and Thornton, J.M., 1993. PROCHECK: a program to check the stereochemical quality of protein structures. Journal of applied crystallography, 26(2), 283-291. https://doi.org/10.1107/S002188989200 9944

NRC, 2011. Nutrient requirements of fish and shrimp. Washington D.C: National Academies Press.

Ozawa, K. and Laskowski, M., 1966. The reactive site of trypsin inhibitors. Journal of Biological Chemistry, 241(17), pp.3955-3961.

Pettersen, E.F., Goddard, T.D., Huang, C.C., Couch, G.S., Greenblatt, D.M., Meng, E.C. and Ferrin, T.E., 2004. UCSF Chimera - a visualization system for exploratory research and analysis. Journal of computational chemistry, 25(13), 1605-1612. https://doi.org/10.1002/jcc.20084treatm ents. Viruses, 8(1), 23. https://doi.org/10.3390/v8010023

Rahman, M.M., 2015. Role of common carp (Cyprinus carpio) in aquaculture production systems. Frontiers in Life Science, 8(4): 399-410.

Rawlings, N.D. and Barrett, A.J., 1994. Families of serine peptidases. In Methods in enzymology (Vol. 244, pp. 19-61). Academic Press.

Schreiber, G., Haran, G. and Zhou, H.X., 2009. Fundamental aspects of proteinprotein association kinetics. Chemical reviews, 109(3), pp.839-860.

Smirnoff, P., Khalef, S., Birk, Y., Applebaum, S.W., 1976. A trypsin and chymotrypsin inhibitor from chickpeas (Cicer arietinum). Biochemical Journal 157: 745-751.

SOFIA, 2016. The State of World Fisheries and Aquaculture. Contributing to Food Security and Nutrition for All. Rome, $20 \mathrm{pp}$.

Song, H.K. and Suh, S.W., 1998. Kunitz-type soybean trypsin inhibitor revisited: refined structure of its complex with porcine trypsin reveals an insight into the interaction between a homologous inhibitor from Erythrinacaffra and tissue-type plasminogen activator. Journal of molecular biology, 275(2), pp.347-363.

Tacon, A. G. J., and Metian, M., 2008. Global overview on the use of fish meal and fish oil industrially compounded aquafeeds: Trends and future prospects. Aquaculture, 285, 146- 158.

Van Zundert, G.C.P., Rodrigues, J.P.G.L.M., Trellet, M., Schmitz, C., Kastritis, P.L., Karaca, E., Melquiond, A.S.J., van Dijk, M., De Vries, S.J. and Bonvin, A.M.J.J., 2016. The HADDOCK2. 2 web server: user-friendly integrative modeling of biomolecular complexes. Journal of molecular biology, 428(4), pp.720-725.

Vangone, A. and Bonvin, A.M., 2015. 
Contacts-based prediction of binding affinity in protein-protein complexes. Elife, 4, p.e07454.

Watanabe, T., 2002. Strategies for further development of aquatic feeds. Fisheries Science, 68,242- 252.

Waterhouse, A., Bertoni, M., Bienert, S., Studer, G., Tauriello, G., Gumienny, R., Heer, F.T., de Beer, T.A.P., Rempfer, C., Bordoli, L. and Lepore, R., 2018. SWISS-MODEL: homology modelling of protein structures and complexes. Nucleic acids research, 46(W1), W296-W303. https://doi.org/10.1093/nar/gky427

Xue, L.C., Rodrigues, J.P., Kastritis, P.L., Bonvin, A.M. and Vangone, A., 2016. PRODIGY: a web server for predicting the binding affinity of protein-protein complexes. Bioinformatics, 32(23), pp.3676-3678.

\section{How to cite this article:}

Gulshan Kumar, Munish Kumar, Gyandeep Gupta and Ranjeeta Kumari. 2019. Computational Interaction Analysis of Cyprinus carpio Trypsin1 and Kunitz Type Soybean Protease Inhibitor. Int.J.Curr.Microbiol.App.Sci. 8(06): 3160-3171. doi: https://doi.org/10.20546/ijcmas.2019.806.378 